\title{
Avaliação da importância do exame clínico para os alunos do curso de graduação da Faculdade de Odontologia de São José dos Campos - UNESP
}

Glauber Marsi*, Ana Cristina Mengue*, Fernanda Bertini**, Luiz Antônio Guimarães Cabral***, Janete Dias Almeida***

\author{
* Cirurgiões-Dentistas, Faculdade de Odontologia de São José dos \\ Campos, FOSJC-UNESP \\ ** Cirurgiã-Dentista, Mestre e Aluna do Curso de Pós graduação, \\ nível Doutorado, FOSJC-UNESP, Programa de Pós Graduação em \\ Biopatologia (Departamento de Biociências e Diagnóstico Oral) \\ *** Professores Adjuntos da Disciplina de Semiologia, FOSJC-UNESP, \\ Programa de Pós Graduação em Biopatologia (Departamento de \\ Biociências e Diagnóstico Oral)
}

\section{RESUMO}

O exame físico e a anamnese são etapas primordiais, além de fundamentais em qualquer área de saúde, visando diagnósticos precisos e tratamentos adequados. O objetivo desse estudo foi avaliar a importância dada pelos alunos, quanto ao processo diagnóstico. 150 alunos voluntários foram avaliados através de um questionário, considerando a importância dada ao exame físico pormenorizado e à anamnese. Os resultados foram analisados por estatística descritiva. 45,6\% dos voluntários deixaram de fazer algum tipo de pergunta; $3,88 \%$ dos alunos do Noturno e $26,21 \%$ do Integral não costumam explicar aos seus pacientes a importância da anamnese; sendo que $66,99 \%$ dos alunos costumam avaliar o sistema estomatognático como um todo; e somente $47,57 \%$ destes sentem-se motivados a realizar o exame físico extra e intrabucal completo nas clínicas. Frente aos resultados verifica-se a necessidade de novos estudos utilizando outros métodos de avaliação, incluindo avaliações da prática clínica, para o aperfeiçoamento do processo ensinos/ aprendizado.

\section{DESCRITORES}

Exame Clínico. Anamnese. Diagnóstico. Pesquisa.
$\Delta$ observação de algum indício de doença (sinal e/ ou sintoma) é o que leva o paciente, na maioria dos casos, a procurar um profissional da saúde (Kerr et al.,1977; Yellowitz et al.,1998; Smith et al., 2000; Tommasi, 2002), cabendo a este último a responsabilidade quanto a elucidação do diagnóstico, aplicação dos recursos terapêuticos pertinentes e acompanhamento do paciente (Kerr et al.,1977; Zegarelli et al., 1978; Yellowitz et al.,1998; Smith et al., 2000; Tommasi, 2002)

No que tange ao diagnóstico, ou a elaboração do diagnóstico diferencial, é de suma importância que o profissional atenha-se ao exame clínico, não só quanto as alterações físicas presentes, bem como à anamnese, valorizando também a história médica (Kerr et al.,1977; Amiel et al.,1997; Smith et al., 2000; Mossey et al.,2001; Tommasi, 2002).

Nos casos de alterações nas estruturas bucais e/ ou anexas, cabe ao cirurgião dentista a identificação e o diagnóstico (Kerr et al.,1977; Yellowitz et al.,1998; Smith et al., 2000; Mossey et al.,2001; Burzynski et al.,2002; Tommasi, 2002).

O exame clínico das estruturas do complexo maxilo-mandibular e anexos deve ser sistemático, ordenado e completo, possibilitando averiguar a presença de alterações nos tecidos duros e moles (Atterbury e Vazirani, 1968; Smith et al., 2000; Tommasi, 2002). 
Deve o paciente ser conscientizado da necessidade de exames clínicos realizados em espaço máximo de um ano, para permitir a identificação precoce de processos patológicos que venham a se desenvolver (Burzynski et al., 2002; Pedroni et al., 2003).

O exame clínico está afeito a dois procedimentos: a anamnese e o exame físico. A anamnese é composta pela identificação do paciente, da queixa principal, da história da doença atual, da história buco-dental, da história médica, dos antecedentes familiares e dos hábitos nocivos e de higiene (Kerr et al.,1977; Zegarelli et al., 1978; Mitchell et al., 1978; Coleman and Nelson,1993; Yellowitz et al.,1998; Smith et al., 2000; Tommasi, 2002).

O exame físico é composto pelo aspecto geral do paciente, desde a primeira impressão, e os exames intra e extra-bucais (Kerr et al.,1977; Mitchell et al., 1978; Coleman and Nelson,1993;;Yellowitz et al.,1998; Smith et al., 2000; Tommasi, 2002).

A anamnese (ana + amnese) é uma palavra de origem grega, que basicamente significa: "o que não pode ser esquecido". Esse processo tem início a partir da avaliação da queixa principal, se presente, e evolui paralelamente às observações clínicas presentes. $\mathrm{Na}$ prática, a anamnese deve ser encarada como a história evolutiva da doença, desde as suas manifestações prodrômicas (manifestações iniciais ou precursoras de uma doença ou crise) até o estágio em que se encontra o paciente (Smith et al., 2000). Na realização da mesma, o paciente pode referir dados que levem a suspeitar da concomitância de outras patologias, de ordem local ou sistêmicas (Atterbury and Vazirani, 1968; Coleman and Nelson,1993; Smith et al., 2000; Burzynski et al.,2002).

$\mathrm{O}$ exame físico pode ou não preceder a anamnese, e tem por objetivo a pesquisa de sinais, que indiquem a presença de alguma alteração patológica, realizado por manobras clássicas, através dos sentidos, a saber: inspeção visual, palpação, percussão, auscultação e olfato (Kerr et al.,1977; Zegarelli et al., 1978; Coleman and Nelson,1993; Burzynski et al.,2002; Smith et al., 2000; Tommasi, 2002). Dessas manobras, considerando-se o complexo maxilo-mandibular, a inspeção visual, direta ou indireta, e a palpação digital são as mais utilizadas (Atterbury and Vazirani, 1968; Mitchell et al., 1978; Smith et al., 2000).

Na primeira impressão, ou seja, quando profissional e paciente têm o primeiro contato, são observados: características físicas, tais como o tamanho corporal, as proporções anatômicas, a forma facial, a postura, os movimentos (a maneira como caminha, por exemplo) e outras características que o cirurgiãodentista julgar importantes, além da observação da idade aparente, do sexo, da raça, bem como características psico-emocionais do paciente (Coleman e Nelson, 1993; Nicodemo e Rode, 2002; Tommasi, 2002). No exame extra-bucal devem ser observados pele da face, do pescoço, dos lábios, dos maxilares, presença de linfonodos palpáveis da região e aspectos das glândulas salivares maiores quanto à cor, à forma / simetria e à consistência: (Atterbury and Vazirani, 1968; Kerr et al.,1977; Mitchell et al., 1978; Coleman and Nelson,1993; Yellowitz et al.,1998; Smith et al., 2000).

No exame intra-bucal devem ser observados mucosa labial e da bochecha, palato mole e duro, orofaringe, soalho da boca, língua, gengiva, dentes, e rebordo alveolar quanto à cor, à forma / simetria e à consistência: (Atterbury and Vazirani, 1968; Kerr et al.,1977; Mitchell et al., 1978; Zegarelli et al., 1978; Coleman and Nelson,1993; Yellowitz et al.,1998; Smith et al., 2000; Tommasi, 2002).

O registro de todas as impressões obtidas através da realização da anamnese e exame clínico apurado fazem parte do processo diagnóstico, o que é esperado em propedêutica estomatológica, que culmina no entendimento sistêmico do processo. Ainda, é um instrumento legal de relevância, sendo, portanto, uma fonte segura para obtenção de dados estatísticos epidemiológicos que auxiliam para conhecer melhor a história natural das doenças (Burzynski et al.,2002; Tommasi, 2002; Pedroni et al.,2003; Jünger et al.,2005) .

Tendo em vista que o exame físico objetivo e a anamnese corresponderem à etapa primordial (Amiel et al.,1997; Mossey et al.,2001; Burzynski et $a l ., 2002)$, além de serem fundamentais em qualquer atendimento na área de saúde, este trabalho visa avaliar o grau de aprendizado e a importância dada pelos alunos (Burzynski et al.,2002; McCann et al.,2005; Schoonheim-Klein et al.,2005), que já adquiriram conhecimento quanto ao processo diagnóstico, procurando observar se os mesmos vêm efetivamente praticando o que foi transmitido (Mossey et al.,2001; Burzynski et al.,2002; Jünger et al.,2005; McCann et al.,2005; Schoonheim-Klein et al., 2006). Tal avaliação justifica-se à medida que profissionais graduados não realizam exame clínico pormenorizado, o que pode levar a diagnósticos imprecisos e tratamentos inadequados (Thompson and Boyle, 1996; Amiel et al.,1997; Burzynski et al.,2002; Jünger et al.,2005; McCann et al.,2005; Schoonheim-Klein et al., 2006;). A reflexão quanto ao processo ensino / aprendizagem é neces- 
sária para o aperfeiçoamento do mesmo (Boone et al., 2001; Mossey et al.,2001; Remmen et al.,2001; Burzynski et al.,2002; Zartman et al.,2002; Macluskey et al.,2004; Jünger et al.,2005; Schoonheim-Klein et al., 2005; Schoonheim-Klein et al., 2006) .

\section{MATERIAL E MÉTODO Voluntários}

Os alunos do $3^{\circ}$ e $4^{\circ}$ ano do Curso Integral e $4^{\circ} \mathrm{e}$ $5^{\circ}$ do Curso Noturno da Faculdade de Odontologia de São José dos Campos - UNESP foram convidados a participar como voluntários desta pesquisa, considerando-se que os mesmos já haviam adquirido conhecimento do processo diagnóstico. Os voluntários que aceitaram participar da pesquisa foram informados sobre os objetivos do estudo e assinaram o Termo de Consentimento Livre Esclarecido. O trabalho de pesquisa ora apresentado foi aprovado pelo Comitê de Ética da Faculdade de Odontologia de São José dos Campos - UNESP, sob protocolo 035/04-PH/ CEP, em anexo.

\section{Questionário}

Os voluntários responderam a um questionário (Anexo A), de 13 perguntas sobre o processo diagnóstico, sendo que cinco perguntas eram de múltipla escolha, sete passíveis de resposta "sim" ou "não" juntamente com um espaço para a justificativa da resposta e apenas uma questão dissertativa.

\section{Análise dos Dados}

Os dados foram analisados usando a distribuição de freqüência das respostas obtidas dos questionários pelo programa computacional MINITAB (versão $12.0,1998)$. Foram analisadas as proporções das respostas obtidas, levando em conta o ano e o período que o aluno está cursando. Consideramos o grau de importância dado ao exame físico pormenorizado e à anamnese pelos alunos participantes da pesquisa, de acordo com as respostas representativas inerentes ao processo diagnóstico.

\section{RESULTADOS}

Dos 150 questionários distribuídos para os alunos voluntários, foram respondidos 103, dos quais 77 $(74,76 \%)$ eram do Curso Integral e apenas 26 $(25,24 \%)$ do Curso Noturno.

Quanto à importância de se fazer uma anamnese completa, todos responderam positivamente. Quando foi perguntado se o aluno deixou de fazer algum tipo de pergunta durante a anamnese por se sentir constrangido ou por não achar importante, 47 alunos $(45,6 \%)$ entre os Cursos Integral e Noturno responderam sim.

Quanto aos itens que seriam importantes conter na ficha clínica, todos tiveram aprovação maior ou igual a 93,2\% (96 alunos entre os Cursos Integral e Noturno).

Foi relatado por 1 aluno do Curso Integral e 1 aluno do Curso Noturno (correspondentes a 1,94\%) que houve 1 paciente que se recusou a responder a toda a anamnese; e 6 alunos do Curso Integral e apenas 1 aluno do Curso Noturno (correspondentes a $6,79 \%$ ) tiveram pacientes que se recusaram a responder alguma pergunta específica da anamnese. Não costumam explicar aos seus pacientes porque a anamnese deve ser feita 4 alunos do Curso Noturno $(3,88 \%)$ e 27 alunos do Curso Integral (26,21\%).

Quando questionado se o aluno considera importante um exame físico apurado com exame intra e extrabucal, 103 alunos (100\%) responderam sim.

Quanto ao exame físico extrabucal foi observado que apenas 61 alunos $(59,22 \%)$ consideram a palpação da glândula parótida importante, e 103 alunos $(100 \%)$.consideram a aferição da pressão arterial importante.

Cerca de cinqüenta e cinco alunos $(53,39 \%)$ responderam que não conseguem realizar adequadamente algumas etapas do exame físico extrabucal, sendo que a palpação de linfonodos e da glândula parótida são as menos executadas durante o exame físico $(57,28 \%$ e $87,37 \%$ respectivamente).

Em relação ao exame físico intrabucal, foi constatado que 75 alunos $(72,81 \%)$ não observam a região da orofaringe, 39 alunos $(37,86 \%)$ não realizam e sondagem periodontal e 37 alunos $(35,92 \%)$ não observam o desenvolvimento da oclusão; valores menores ou iguais a 13,59\% foram encontrados em relação a não observância dos seguintes itens: mucosa labial $(7,76 \%)$, mucosa oral $(3,88 \%)$, palato duro $(3,88 \%)$, palato mole $(12,62 \%)$, soalho da boca $(13,59 \%)$, língua $(1,94 \%)$, gengiva $(1,94 \%)$, dentes $(1,94 \%)$ e rebordo alveolar $(9,70 \%)$.

Em relação à motivação dos alunos em realizar o exame físico extra e intrabucal completo nas clínicas, somente 47,57\% (37 alunos do Curso Integral e 12 alunos do Curso Noturno) responderam sim.

\section{DISCUSSÃO}

Burzynski, et al., 2002 afirmam que ainda existem grandes deficiências quanto ao exame físico na prevenção e controle do câncer bucal, o que mostra a 


\section{ANEXO A - Questionário}

01) Você considera importante fazer a anamnese?

( ) $\operatorname{Sim}$ ( ) Não

Por quê:

02) Marque um $X$ nos itens que você considera ser importante na ficha clínica:
( ) História médica
( ) História da doença atual
( ) História buco-dental
( ) História médica familiar
( ) Hábitos nocivos e higiênicos

03) Durante a anamnese você já deixou de fazer algum tipo de pergunta ao paciente por se sentir constrangido ou por não achá-la importante?

( ) $\operatorname{Sim}($ ) Não

04) Algum paciente que você estava atendendo já se recusou a responder:

- a toda a anamnese? ( ) Sim ( ) Não

- a alguma pergunta específica da anamnese?

( ) $\operatorname{Sim}$ ( ) Não

Qual:

05) Você costuma explicar ao paciente porque a anamnese deve ser feita?

( ) $\operatorname{Sim}$ ( ) Não

06) Você considera importante um exame físico apurado com exame extrabucal (palpação de linfonodos e da glândula parótida, observação do aspecto geral da face) e exame intrabucal (observação completa e minuciosa dos tecidos moles e duros)?

( ) $\operatorname{Sim}$ ( ) Não

Por quê:

07) Marque um $X$ nos itens que você considera importante em relação ao exame físico extrabucal:

( ) Verificação do pulso

( ) Verificação da pressão arterial

( ) Observação do aspecto geral da face, cabeça e pescoço

( ) Palpação de linfonodos

( ) Palpação das glândulas salivares maiores

08) Você consegue realizar adequadamente os procedimentos do exame físico extrabucal? Caso responda não, especifique qual o procedimento:

( ) $\operatorname{Sim}$ ( ) Não
Qual:

09) Qual desses passos abaixo você realiza durante o exame físico extrabucal nas clínicas da faculdade?

( ) Observação dos aspectos gerais do paciente (ex: porte físico, deambulação, alterações de pele, etc)

( ) Palpação de linfonodos

( ) Palpação da glândula parótida

( ) Verificação do pulso

( ) Verificação da pressão arterial

10) Marque um $X$ nos itens que você considera importante observar em relação ao exame físico intrabucal:

( ) Mucosa labial

( ) Demais mucosas de revestimento bucal

( ) Palato duro

( ) Palato mole

( ) Soalho da boca

( ) Língua

( ) Gengiva

( ) Dentes

( ) Profundidade de sulco

( ) Aspectos oclusais

( ) Rebordo alveolar

( ) Orofaringe

11) Você costuma avaliar o sistema estomatognático como um todo quando você realiza o exame físico intrabucal numa disciplina específica da odontologia (por exemplo: dentística, endodontia, próteses...)?

( ) Sim ( ) Não

12) Qual dos itens abaixo você não costuma observar com freqüência durante os exames físicos intrabucais?

( ) Mucosa labial

( ) Mucosas de revestimento bucal

( ) Palato duro

( ) Palato mole

( ) Orofaringe

( ) Soalho da boca

( ) Língua

( ) Gengiva

( ) Dentes

( ) Profundidade de sulco

( ) Aspectos oclusais

( ) Rebordo alveolar

13) Você se sente motivado a realizar o exame físico extra e intrabucal completo nas clínicas?

( ) $\operatorname{Sim}$ ( ) Não

Se não, por quê? relevância da pesquisa realizada. Considerando que o processo ensino / aprendizagem deve ser implementado, é necessário conhecer as deficiências para a busca de metodologia que possa minimizá-las.
Apesar de todos os alunos terem respondido que consideram importante realizar a anamnese, alunos do Cursos Integral e Noturno relataram que deixaram de fazer algum tipo de pergunta durante a anamnese 
por se sentirem constrangidos ou por não acharem importante. Imprescindível salientar que alguns alunos do Curso Noturno e Integral não costumam explicar aos seus pacientes porque a anamnese deve ser feita. Do mesmo modo, foi verificado por Amiel, et al.,1997, que $(75,1 \%)$ dos alunos entrevistados não realizam adequadamente a anamnese. Em (2005) Jünger, et al., observaram que a execução inapropriada da anamnese acarreta uma relação profissional / paciente pobre em confiança, concluindo que os mesmos alunos apresentam dificuldades quanto às técnicas clínicas para o diagnóstico.

Embora todos os alunos tenham respondido que consideram importante realizar um exame físico apurado, com palpação de linfonodos, da glândula parótida, observação do aspecto geral da face e observação completa e minuciosa dos tecidos moles e duros intrabucais, cerca de $(53,39 \%)$ responderam que não conseguem realizar adequadamente algumas etapas do exame físico extrabucal. Ainda, em relação ao exame físico extrabucal foi obtido que $(87,37 \%)$ dos alunos, apesar de responderem que realizam a palpação da glândula parótida, $(59,22 \%)$ não consideram esta etapa importante, o que se torna relativamete contraditório.

Fato incomum foi observado durante a análise dos questionários quando alunos responderam que não costumam observar nem gengivas nem dentes durante o exame clínico intrabucal, o que pode ser devido a uma falta de atenção por parte destes durante a interpretação do mesmo, visto que geralmente estes dois ítens são os mais avaliados durante uma consulta.

Quando questionado se o aluno costuma avaliar o sistema estomatognático como um todo, na realização do exame físico intrabucal numa disciplina específica da odontologia (por exemplo: dentística, endodontia, prótese, etc.), $66.99 \%$ responderam sim, porém somente $47,57 \%$ se sentem motivados em realizá-los de maneira completa nas clínicas. Schoonheim-Klein, et al.,2006 observaram que $81,3 \%$ justificativam a não realização de exame completo baseadas na falta de tempo disponível, falta de incentivo por parte dos docentes e fichas clínicas incompletas. À medida que muitos docentes têm um tempo reduzido para o desenvolvimento pleno e necessário entre teoria e clínica, muitos acabam se dedicando a uma anamnese e exame clínico mais específico à disciplina em que atuam, o que leva a uma ficha clínica muitas vezes incompleta sem perguntas e quesitos suficientes para que se tenha uma visão abrangente do estado de saúde do paciente. Os trabalhos de Bo- one, et al.,2001 e Zartman, et al.,2002 revelam que algumas vezes os membros do corpo docente menosprezam a importância do processo diagnóstico em todas as clínicas o que leva a não realização de determinados procedimentos.

Remmen, et al.,2001 relataram que alunos que receberam uma educação reformulada submetidos à avaliação, obtiveram melhores resultados em relação àqueles que foram educados pelos métodos tradicionais. Jünger, et al., 2005 verificaram que alunos acompanhados de maneira mais intensa pelo professor acabam por obter melhor desempenho na comunicação durante a anamnese.

Macluskey, et al., 2004 e Schoonhein-Klein, et al., 2005 propuseram que seja utilizada uma metodologia unificada de avaliação entre os docentes, evitando dessa forma diferentes resultados em relação a um único ponto, visto que a concepção do aprendizado dos alunos é influenciada pelo método de utilizado (Schoonhein-Klein, et al.,2006).

Baseados nos resultados dessa pesquisa, observouse que a anamnese e o exame clínico pormenorizado ainda não são realizados de maneira adequada, muitas vezes devido a uma dificuldade por parte dos alunos, por falta de tempo disponível para a realização do mesmo, falta de incentivo por parte dos docentes e muitas vezes por uma ficha clínica incompleta. Métodos de avaliação longitudinal da performance e evolução clínica individual e em grupo dos alunos em processo de graduação devem ser realizados, visando identificar, reformular e corrigir as áreas deficientes, como foi proposto por Boone, et al.,2001, Mossey, et al.,2001, Remmen, et al.,2001 e Zartman, et al.,2002, Jünger, et al.,2005, McCann, et al.,2005.

\section{CONCLUSÃO}

Considerando as limitações do estudo, é possível concluir que são necessários novos estudos utilizando outros métodos de avaliação, incluindo prática clínica, para o aperfeiçoamento do processo ensino/ aprendizado.

\section{ABSTRACT}

\section{Evaluation of the importance of clinical examination for the undergraduate course students at São José dos Campos School of Dentistry - UNESP}

Physical examination and anamnesis are foremost and fundamental in any health area in order to obtain precise diagnosis and suitable treatment. The objective of this study was to evaluate the importance given 
by the students to the diagnosis process. A total of 150 volunteer students were evaluated through a questionnaire taking into consideration the importance given to detailed physical examination and anamnesis. The results were analyzed by descriptive statistics: $45.6 \%$ of the volunteers failed to ask some kind of question; $3.88 \%$ of the students from the night course and $26 \%$ from the comprehensive course are not used to explaining the importance of anamnesis to their patients; $66.99 \%$ of the students are used to evaluating the stomatognathic system as a whole; and only $47.57 \%$ of the students feel motivated to perform the complete extra- and intra-oral physical examination in the clinic. In view of the results, we can see that there is a need for new studies using other evaluation methods, including evaluation of clinical practice, in order to improve the teaching/learning processes.

\section{DESCRIPTORS}

Clinical Examination. Anamnesis. Diagnosis Research.

\section{REFERÊNCIAS}

1. Amiel, GE.; Tann, M.; Krausz, MM.; Bitterman, A.; Cohen, R. Increasing examiner involvement in an objective structured clinical examination by integrating a structured oral examination. The American Journal of Surgery 1997; 173:546-49.

2. Atterbury, RA.; VaziraniA, SJ. Examination procedure for oral cancer. Oral Surgery, Oral Medicine and Oral Pathology 1968; 26(1):80-6.

3. Boone, WJ.; Mcwhorter, AG.; Seale N.S. Purposeful assessment techniques (PAT) applied to an OSCE-based measurement of competencies in pediatric dentistry curriculum. Journal of Dental Education 2001;.65(11):1232-37.

4. Burzynski, NJ.; Rankin, KV.; Silverman Jr, S.; Scheetz, JP.; Jones, DL. Graduating Dental Students' perceptions of oral cancer education: results of an exit survey of seven dental schools. Journal Of Cancer Education 2002; 17(2): 83-4.

5. Coleman, GC.; Nelson, JF. Principles of Oral Diagnosis. St Louis. Mosby Year Book, 1993.

6. Jünger, J.; Schäfer, S.; Roth, C.; Schellberg, D.; Ben-David, MF.; Nikendel, C. Effects of basic clinical skills training on objective structured clinical examination performance. Medical Education 2005;39:1015-20.

7. Kerr, DA.; Ash, MM.; Millard, HD. Diagnóstico Oral-4a ed. Rio de Janeiro: Guanabara Koogan, 1977.

8. Macluskey, M.; Hanson, C.; Kershaw, A.; Wight, AJ.; Ogden, GR. Development of a structured clinical operative test (SCOT) in the assessment of practical ability in the oral surgery undergraduate curriculum. British Dental Journal 2004; 197(2): 60.

9. Mccann, PJ.; Sweeney, MP.; Gibson, J.; Bagg, J. Training in oral disease, diagnosis and treatment for medical students and doctors in the United Kingdom. Br J Oral Maxillofac Surg. 2005; 43(1):61-4.

10. Mitchell, DF.; Standish, SM.; Fast, TB. Oral Diagnosis/Oral Medicine $-3^{\text {rd }}$ ed. Philadelphia: Lea \& Febiger, 1978.

11- Mossey, PA.; Newton, JP.; Stirrups, DR. Scope of the OSCE in the assessment of clinical skills in dentistry. British Dental Journal 2001;190(6):.323-26.

12. Nicodemo, D.; Rode, SM. Orientações psicológicas para o manejo clínico dos pacientes com indicação de prótese ocular. RPG - Revista de Pós-Graduação 2002;.9(3,):224-31.

13. Pedroni, CR.; de Oliveira, AS.; Guaratini, MI. Prevalence study of signs and symptoms of temporomandibular disorders in university students. Journal of Oral Rehabilitation 2003;30(3):283-89.

14. Remmen, R.; Scherbier, A.; Van der Vleuten, C.; Denekens, J. Effectiveness of basic clinical skills training programmes: A cross-sectional comparison of four medical schools. Medical Education 2001;.35:121-28.

15. Schoonhein - Klein, M.; Habets, L.; Aarman, IHA; Van der Vleuten, CP.; Hoogstraten, J.; Van der Velden, U. Implementing an objective structured clinical examination (OSCE) in dental education: effects on students' learning strategies. European Journal of Dental Education 2006;10:226-35.

16. Schoonhein-Klein, M.; Walmsley, AD.; Habets, L.; Van der Velden, U.; Manogue, M. An implementation strategy for introducing an OSCE into a dental school. European Journal of Dental Education 2005;9:143-49.

17. Smith, RA.; Mettlin, CJ.; Davis, KJ.; Eyre, H. American Cancer Society Guidelines for the early detection of cancer. CA-A Cancer Journal for Clinicians 2000;50(1):34-49.

18. Thompson, PJ.; Boyle, CA. Auditing Clinical Teaching In Oral Surgery: The use of a student log book. Dental Up date, 1996:23(7):283-86.

19. Tommasi, AF. Diagnóstico em Patologia Bucal- $3^{\text {a }}$ ed. São Paulo: Pancast, p.25-42 2002.

20. Yellowitz, J. Knowledge, opinions and practices of general dentists regarding oral cancer: A pilot survey. The Journal of The American Dental Association 1998; 129(5):579- 83.

21. Zartman, MR.; Mcwhorter AG.; Seale, NS.; Boone, J.W. Using OSCE-based evaluation: curricular impact over time. Journal of Dental Education 2002; 66(12):1323-30.

22. Zegarelli, EV.; Kutscher, AH.; Hyman, GA. Diagnosis of Diseases of Mouth and Jaws $-2^{\text {nd }}$ ed. Philadelphia: Lea \& Febiger, 1978.

Recebido em 07/04/2009 Aceito em 29/06/2009 